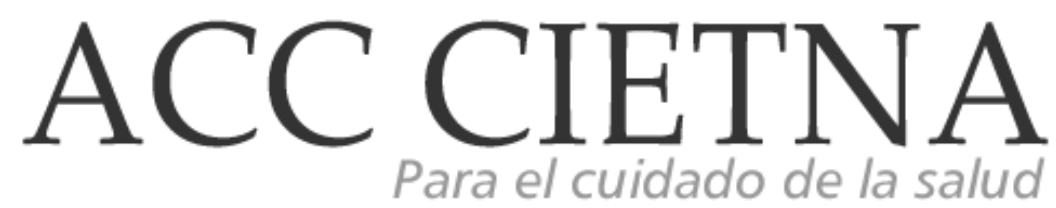

https://doi.org/10.35383/cietna.v6i2.271

\title{
Percepción de las personas cuidadoras en relación al programa de atención domiciliaria para adultos mayores ESSALUD, 2016
}

Dávila Dávila Gloria Liset ${ }^{1}$, Pinzón Dávila Rocío del Pilar², Saavedra Covarrubia Mirian Elena ${ }^{3}$

\begin{tabular}{l} 
INFORMACIÓN DEL ARTÍCULO \\
\hline Historia del artículo: \\
Recibido el 30 de septiembre de 2019 \\
Aceptado el 22de noviembre de 2019 \\
\hline Palabras clave: \\
Percepción \\
Cuidadoras \\
Atención domiciliaria \\
Adulto mayor \\
\hline
\end{tabular}

\section{RESUMEN}

La presente investigación científica es de naturaleza cualitativa, con abordaje de estudio de caso, titulada: "Percepción de las personas cuidadoras en relación al programa de atención domiciliaria para adultos mayores ESSALUD, 2016"; que tuvo como objetivos: describir, analizar y comprender la percepción de las personas cuidadoras en relación al Programa de Atención Domiciliaria (PADOMI) para adultos mayores. Los sujetos de estudio fueron 13 personas cuidadoras de adultos mayores inscritas en el Programa de Atención Domiciliaria (PADOMI). El escenario donde se realizó la investigación fueron los hogares de los adultos mayores, que son atendidos por personas cuidadoras. Durante todo el desarrollo de la investigación, se consideró los criterios éticos de Sgreccia y los criterios de rigor científico. Se utilizó el análisis de contenido temático, emergiendo las siguientes categorías: Escasa participación de la enfermera durante la atención domiciliaria del paciente; Limitaciones organizacionales para el cumplimiento de la atención domiciliaria; Rutinización del equipo de salud durante la visita domiciliaria; Percepción de la atención domiciliaria por parte de los familiares.

\footnotetext{
'Licenciada en Enfermería del Hospital Público de Chota, Cajamarca, Perú. Email: gloriali_davila@hotmail.com ¿Licenciada en Enfermería del área de Salud Ocupacional en Universidad Católica Santo Toribio de Mogrovejo, Chiclayo, Perú. Email: shiopinzon.24@hotmail.com

${ }_{3}^{3}$ Doctora en Enfermería. Docente de la Escuela de Enfermería - Universidad Católica Santo Toribio de Mogrovejo, Chiclayo, Perú. Email: msaavedra@usat.edu.pe. ORCID: https://orcid.org/0000-0002-8139-3816
} 
Perception of caregivers in relation to the adult care program for senior adults ESSALUD, 2016

ABSTRACT

Keywords:

Perception

Caregivers

Home

Care

Elderly
The present scientific research is qualitative, with a case study approach, entitled: "Perception of caregivers in relation to the home care program for oler adults ESSALUD, 2016"; Which aimed to: describe, analyze and understand the perception of caregivers in relation to the Home Care Program (PADOMI) for older adults. The study subjects were 13 elderly caregivers enrolled in the Home Care Program (PADOMI). The scenario where the research was carried out were the homes of the elderly, who are cared for by carers. Throughout the development of the research, we considered the ethical criteria of Sgreccia and the criteria of scientific rigor. The thematic content analysis was used, with the following categories emerging: Poor participation of the nurse during the home care of the patient; Organizational limitations for compliance with home care; Routinization of the health team during the home visit; Perception of home care by family members.

\section{Introducción}

La Organización Mundial de la Salud (OMS), señala que estamos presenciando una verdadera revolución demográfica en el mundo, dado que la población adulta mayor está creciendo a un ritmo sin precedentes, como resultado de la mejora de las condiciones de vida y los avances de la ciencia médica. Así mismo la OMS avizora que para el año 2025, esta población se duplicará, lo que plantea hoy en día retos al Estado y a la sociedad en su conjunto para atender las crecientes demandas de este grupo poblacional, que debe prever cómo refuerza sus sistemas de protección social para estar en capacidad de atenderlas adecuadamente ${ }^{1}$. Así mismo, durante las últimas décadas la disminución mundial de la natalidad y la mortalidad ha llevado progresivamente al incremento de la población adulta mayor. En el Perú el ritmo de crecimiento poblacional se viene incrementando desde hace tres décadas, se calcula que para fines del 2025 la población adulta mayor representará el 7.68\% de la población nacional y en el 2025 representará el 13.27\%. Esto significa que en los próximos 20 años este grupo poblacional llegará casi a duplicarse².

Con esto enfatizamos, que el envejecimiento está ascendiendo en nuestra población; ya que esto se debe al aumento de las enfermedades $y$ discapacidades que tienen las personas, por lo cual consideramos que la mayoría de las personas adultas mayores no acuden a los servicios hospitalarios. Esto indica que fue importante realizar esta investigación de tesis enfocando al área de PADOMI, puesto que se observó las dificultades que tienen los cuidadores respecto al cuidado, ya que estas personas no están capacitadas debidamente.

En este sentido, las necesidades de salud de los adultos mayores son diferentes de las otras etapas de vida, por ser un grupo poblacional complejo donde confluyen por un lado aspectos intrínsecos 
del envejecimiento, alta prevalencia de enfermedades crónico degenerativas, mayor frecuencia de alteraciones en la funcionalidad o dependencia física, que lo sitúan en una posición de vulnerabilidad; y con aspectos extrínsecos como la falta de recursos económicos, débil soporte familiar y social, que lo colocan en situación de desprotección, que usualmente generan daños mal diagnosticados o que pasan inadvertidos, contribuyendo al deterioro de la salud de estas personas, esto conlleva a una pérdida de autonomía en los Adultos Mayores (AM), surge entonces la necesidad de cuidado de contar con ayuda de otras personas para satisfacer las demandas; potencial que se desarrolla según la situación a las que se vean enfrentadas las personas 3 .

Es por ello que el Programa de Atención Domiciliaria (PADOMI) de EsSalud es el sistema integral de atención sanitaria en el hogar dirigida a los pacientes asegurados que por alguna discapacidad no puedan acudir al centro asistencial para el manejo y control de sus problemas de salud. Como parte de EsSalud, el programa brinda servicios domiciliarios que incluyen visitas médicas, enfermería, terapia física, servicio social, psicología, atención de urgencias, hospitalización en casa, laboratorio y farmacia ${ }^{4}$.

Sin embargo, los sistemas sanitarios tratan de satisfacer las necesidades de cuidado de salud enfocando su atención en términos de mantenimiento de la capacidad funcional. Cuidar será siempre indispensable para la perpetuidad de la sociedad, es el pilar de la profesión de enfermería, pero también tiene un componente no profesional, el cuidado familiar en el domicilio, que es considerado una obligación moral ${ }^{5}$.

Las investigadoras expresaron que durante las prácticas de pregrado en el 6to ciclo, observaron las dificultades y percepciones que tenían las personas cuidadoras sobre el servicio de PADOMI; quienes manifestaron como problemática la necesidad de contar con material básico para que realicen ellos mismos el cuidado, hicieron mención que la doctora realiza la visita para la programación de sus familiares, una vez al mes; otro punto que comentaron es que la enfermera realiza la visita una vez a la semana, para hacer entrega del material que utilizarán, y también realizaban el control semanal de las personas adultas mayores.

Por otra parte, las cuidadoras comentaron que es conveniente que las enfermeras realicen la visita domiciliaria, aproximadamente 3 veces a la semana; sin embargo, la enfermera del programa de atención domiciliaria realiza la visita 1 vez por semana; así mismo recibir una capacitación para tener mayor conocimiento acerca de los cuidados que realizan las enfermeras. Además, agregaron que necesitan más apoyo del personal de salud, de tal forma que les permita brindar de manera eficaz los cuidados básicos y primordiales a sus pacientes, ya que no siempre cubren las necesidades que ellos requerían para su tratamiento.

\section{Metodología}

El presente trabajo de investigación fue de tipo cualitativo6; siendo así, un proceso activo, sistemático, riguroso; en el cual se toman decisiones sobre lo investigado proporcionando una descripción explícita del fenómeno a estudiar.

Puesto que permitió describir, analizar y comprender la percepción de las personas cuidadoras en relación al Programa de Atención Domiciliaria en adultos mayores; y se tomó al sujeto de estudio como un ser integral, en donde se utilizó como una técnica de recolección de datos la observación y la entrevista. 
En este trabajo de investigación los sujetos con los cuales trabajamos fueron las personas cuidadoras. Así mismo en el Programa de Atención Domiciliara del Hospital Naylamp existe solo una enfermera que realiza la visita domiciliaria y un médico que realiza la visita una vez al mes. Cabe resaltar que este programa de atención domiciliaria, cuenta con un total de 571 personas inscritas; sin embargo, el $10 \%$ de las personas inscritas son atendidas; de acuerdo a las necesidades de los usuarios, eso quiere decir que solo 50 personas son atendidas por el personal de salud en este programa.

Ya que el programa de atención domiciliaria no atiende a todos los inscritos por la poca cantidad de enfermeras que laboran en el programa de atención domiciliaria, es por ello que solo una enfermera no se abastece a realizar las visitas a todos los inscritos, en tanto trabajan con el $10 \%$ siendo una cantidad aceptable, para que una enfermera realice la visita domiciliaria una vez por semana a los adultos mayores.

Los sujetos de investigación fueron las personas cuidadoras encargadas de los adultos mayores inscritos en el Programa de Atención Domiciliaria del Hospital Naylamp. Las cuales fueron protegidas con un código al momento de realizar las entrevistas referentes a las iniciales PC1, PC2, PC3, PC4, PC5, PC6, PC7, PC8, PC9, PC10, PC11, PC12, PC13. La muestra se determinó por los criterios de saturación y redundancia. Por lo cual se consideraron los siguientes criterios de selección.

Después de recopilar la información y las evidencias que fueron necesarias se dio paso al análisis de contenido temático, iniciando la transcripción de las entrevistas manteniendo de forma integral el mensaje que fue expuesto. De ahí que pasaron a discursos y se extrajo la unidad de análisis el cual permitió que se forme las categorías. Finalmente se procedió a analizar las entrevistas y discursos paralelos con el marco teórico, y se concluyó con la formulación de nuevos conocimientos.

El análisis de datos permitió ordenar la información recolectada de modo que se pueda organizar, estructurar, sintetizar e identificar los datos de la investigación. Dentro de las fases para elaborar el sistema de categorías de análisis de contenido tenemos: pre análisis, explotación del material, tratamiento e interpretación de los resultados obtenidos.

La primera fue "pre análisis", es una etapa en donde se organizó el material y se analizó. Las investigadoras en esta fase dispusieron de la información obtenida en las entrevistas que fueron realizadas a las personas cuidadoras, mediante la transcripción del grabado del contenido de la información, igual como se recibió, en tanto, se interpretó la información, además se hizo lectura de la información que se obtuvo de las cuales se hizo observaciones ${ }^{7}$.

La segunda fue "explotación del material", esta fase mencionaba sobre las partes del contenido de los cuales las investigadoras pudimos elaborar los análisis, estableciéndolo como fuente principal que se ajustó a los requerimientos de la investigación. Las investigadoras procedieron a analizar el material obtenido mediante la codificación la cual consiste en "transformar los datos brutos del texto a través de descomposición, agregación y la enumeración para la representación del contenido". Finalmente se agrupó conforme a las similitudes.

La tercera fase fue "tratamiento e interpretación de los resultados obtenidos", es aquí en donde los resultados brutos debieron resultar significativos, y a la vez ser válidos. Finalmente se estableció un código, a los datos que fueron obtenidos por las investigadoras, incluyéndolo en nuestro marco 
teórico y se sustentó para establecer la relación y comparación científica.

Durante la investigación se tuvo en cuenta los criterios de rigor científico, credibilidad, auditabilidad y transferibilidad8; de igual manera los principios éticos, el valor fundamental de la vida y el respeto a su dignidad, principio de la libertad y la responsabilidad, socialización y subsidiariedad 9 .

\section{Resultados, análisis y discusión}

Según el Modelo de atención integral de salud con enfoque familiar y comunitario definido con la reforma de salud, el cuidado domiciliario solo está enfocado a la atención de adultos mayores con dependencia y/o con diagnóstico de enfermedad terminal, donde el principal propósito de esta atención es proporcionar a los cuidadores y a la familia las herramientas necesarias para asumir el cuidado integral de la persona con dependencia, resguardar la continuidad y oportunidad de atención en la red asistencial y mantener un registro actualizado de las personas con dependencia. Sin lugar a dudas, falta mayor desarrollo de la asistencia domiciliaria a nivel comunitario e investigaciones que avalen su uso con los adultos mayores ${ }^{10}$.

Por ello fue importante reflexionar sobre como las cuidadoras describen el trabajo o participación que realiza la enfermera en la atención domiciliaria del Hospital Naylamp como equipo multidisciplinar, a la vez analizar y comprender dicha perspectiva brindada por las propias cuidadoras enfocada a la labor profesional de enfermería. Es así que al analizar los resultados de la presente investigación surgieron categorías, las mismas que permitieron describir, analizar y comprender sus percepciones.

\section{ESCASA PARTICIPACIÓN DE LA ENFERMERA DURANTE LA ATENCIÓN DOMICILIARIA DEL PACIENTE}

Los cuidados de enfermería tienen entonces una importancia vital, tanto por su participación clave en el equipo de salud como por su potencial de respuesta a las necesidades de cuidado de la salud de los adultos mayores en todos los niveles de atención, y en forma especial en la atención primaria, cuidados a largo plazo y en hospitalización domiciliaria donde la enfermera se comporta como el pilar fundamental para lograr la integralidad y continuidad de los cuidados en los ancianos.

En un programa de hospitalización domiciliaria, el profesional de enfermería se destaca por ser el principal sujeto que otorga orientación $y$ transmisión de información al paciente y a la familia, realiza los procedimientos técnicos, coordina el ingreso y egreso de los pacientes al programa, capacita al personal, evalúa las condiciones socioeconómicas de la familia, etcétera. Las funciones que cumple una enfermera son múltiples, complejas, demandan tiempo y significan gran responsabilidad, ya que el paciente, en este contexto de cuidado, no se encuentra bajo la supervisión continua de un equipo de salud como en el hospital ${ }^{10}$.

Así mismo, las cuidadoras por ser sujetos de la investigación, durante las entrevistas realizadas manifestaron que la participación de la enfermera en el programa de atención domiciliaria es muy escasa, debido a las múltiples funciones y responsabilidades que tiene la enfermera y el gran número de población con el que cuenta el programa PADOMI para la atención de la propia enfermera; por ello es importante que la imagen de la enfermera se vea presente, ya que forman parte del equipo de salud y son las que deben velar ante las necesidades de cuidado en salud hacia los adultos mayores, la función de la enfermera debe ser enfocada al cuidado domiciliario, de esta forma se mantiene vigilado el estado de salud en la que se encuentran las personas adultas mayores. Se menciona en los siguientes discursos:

“...No ha venido la enfermera ni para cuando le inscribimos a mi suegro ha PADOMI, nunca vino, solamente vino la asistente social a verificar en que condición está el 
domicilio, a donde vivía y quienes eran las personas que estaban a su cargo quien le daba los cuidados, pero enfermería no ha venido, no sabía de la visita de enfermería..." (PC3)

“...Enfermera no viene, solamente el médico nada más, ninguna enfermera, solo médico, viene mensualmente, terapista tampoco viene..." (PC8)

"...La enfermera viene cuando tiene que cambiarle la sonda lo hace cada 15 días, la cambia, cura las heridas la revisa. La enfermera demora 10min cuando no cambia sonda, solo la revisa y le ve la curación, cuando no le coloca sonda solo viene a revisar las heridas y realiza la limpieza de las úlceras y ella me dice cómo debo de hacerlo, por ejemplo, hoy vino cambió sonda, limpió todo y ya luego me explicó cómo debo de hacerlo yo..." (PC1)

Las investigadoras fueron a realizar entrevista al médico jefe del programa PADOMI, del cual no se obtuvo respuesta directa por no encontrarse de manera permanente en dicho programa y debido a que realiza la visita domiciliaria a los pacientes. Fue la enfermera encargada del servicio quien nos concedió la entrevista, en donde nos manifestó, que la doctora del programa tiene conocimiento del escaso personal de enfermería que hay en el servicio, sin embargo la doctora del servicio no es encargado de la gestión de personal de salud, sino gestión de recursos humanos, a la vez la enfermera manifestó que ella tiene doce años en el servicio siendo de su agrado, pero desde un inicio trabajo sola en dicho servicio, hoy en día la enfermera realiza horas extras en otro servicio, en PADOMI se ha reducido los días de visitas domiciliarias a dos veces por semana, de los que eran tres veces a la semana, para que cumpla en otro servicio con el tiempo de trabajo. Además, algunas veces le piden que cubra horas de enfermería en otros servicios para reemplazar a la enfermera que falta. Es por ello la importancia de aumentar el personal en el servicio de PADOMI para evitar la sobrecarga del trabajo.

La actuación de la enfermera tiene un papel destacado en la atención domiciliaria procurando cuidados y asegurando su continuidad a lo largo de todos los procesos que sufren los pacientes más vulnerables ${ }^{11}$.

Las enfermeras comunitarias en el ámbito domiciliario, valoran de manera individual las necesidades del paciente y sus cuidadores, diseñan planes de intervención negociados con los pacientes para que sean realistas y aplicables dentro de un contexto de prestaciones previamente definidas. Además, utilizan programas de educación para la salud y de entrenamiento también resuelven la coordinación con otros niveles; sistemas y miembros del equipo asistencial en bien de su paciente. Están comprometidas con la búsqueda de los recursos que permitan a su paciente y cuidador gestionar sus propias necesidades, coopera y participa activamente con las organizaciones y redes comunitarias, organizaciones no gubernamentales, asociaciones de ayuda mutua y demás instituciones públicas o privadas que tienen por objetivo mejorar la salud del conjunto de las personas 12

Se considera importante la actuación de enfermería en la atención domiciliaria, porque desde la perspectiva de las cuidadoras, no se evidencia la participación domiciliaria de las enfermeras, muchas de ellas opinaron que no sabían de la participación de enfermería dentro del programa de atención domiciliaria. Sin embargo, enfermería debe destacar por su eficiencia al brindar los cuidados según las necesidades que encuentra en cada visita domiciliaria, por ello se hace necesario ampliar el número de profesionales enfermeros para planificar la programación en las visitas domiciliarias. 


\section{LIMITACIONES ORGANIZACIONALES PARA EL CUMPLIMIENTO DE LA ATENCIÓN DOMICILIARIA}

Reconocer las propias limitaciones es imprescindible para poder avanzar, pero es igualmente importante no crear en nosotros mismos nuevos límites que coarten nuestro propio desarrollo, como no aceptar los nuevos retos y responsabilidades o instalarse en el conformismo derivado del contenido funcional delegado ${ }^{13}$.

En el marco del Día Internacional de la Enfermería, que tiene lugar el 12 de mayo, la Organización Panamericana de la Salud/ Organización Mundial de la Salud (OPS/OMS) Ilamó a formar más enfermeras en América Latina y el Caribe para prestar una asistencia cualificada, y abordar las inequidades en su distribución y su migración ${ }^{14}$.

Su escasez, distribución geográfica desigual y migración, suponen obstáculos para asegurar el acceso y la cobertura de salud para todos.

Muchos países de América Latina y el Caribe enfrentan un déficit de personal de enfermería, lo que supone un obstáculo para que todas las personas de la región tengan acceso y cobertura universal de salud, advirtieron expertos de la OPS/OMS.

Según la OMS, se necesitan alrededor de 23 médicos, enfermeras y parteras cada 10.000 habitantes para brindar servicios esenciales de salud a la población. En las Américas, alrededor del $70 \%$ de los países cuentan con los profesionales necesarios, y en algunos casos los superan, pero enfrentan desafíos en su distribución y formación.

De tal modo, las autoras detallan que las limitaciones organizacionales, son las barreras y limitantes que tiene el programa de atención domiciliaria por el mismo hecho de la falta de profesionales de enfermería, médicos especializados en Geriatría y terapistas; para brindar el cuidado que necesitan aquellos adultos mayores inscritos en dicho programa.
Por otro lado, el Programa de Atención Domiciliaria (PADOMI) de Essalud es el sistema integral de atención sanitaria en el hogar dirigida a los pacientes asegurados que por alguna discapacidad no puedan acudir al centro asistencial para el manejo y control de sus problemas de salud. Como parte de Essalud, el programa brinda servicios domiciliarios que incluyen visitas médicas, enfermería, terapia física, servicio social, psicología, atención de urgencias, hospitalización en casa, laboratorio y farmacia. La población que recibe atención por el programa está compuesta mayoritariamente por adultos mayores con alguna discapacidad, con enfermedades en estadio avanzado o terminales y poshospitalizados ${ }^{15}$.

En definitiva, para nosotras como autoras, el término de Programa de Atención Domiciliaria, es el servicio de salud integral brindado por el hospital para aquellas personas específicamente adultos mayores, que tienen alguna discapacidad, y no pueden realizar las actividades básicas de la vida diaria por sí mismas, que son apoyadas por el personal de salud de dicho programa en sus hogares; con el propósito de recibir los mismos cuidados que en el hospital. Se puede observar en los siguientes discursos:

“...sería bueno que como enfermería es una de las carreras que mucho trabaja con esto del adulto mayor, sería bueno que venga la enfermera siquiera a darle una miradita a sus pacientes que tiene a cargo porque ella es la que organiza el programa ella hace la programación y no sabe con qué paciente están. Yo sugiero que la gerencia o el Hospital Naylamp tome en cuenta en el programa de PADOMI la incrementación de más personal de enfermería porque una sola enfermera que se abastezca para realizar sus visitas poniendo sonda nasogástrica, sonda Foley $u$ otros dispositivos que tengan el paciente es muy tedioso por lo menos tendrá que haber unas 3 enfermeras para que puedan abarcar toda la atención de los ancianos que es una persona que necesita bastante cuidado $y$ 
dedicación aparte de las personas que están a cargo que somos las cuidadoras..." (PC3)

Las investigadoras realizaron la entrevista a la enfermera de PADOMI, en donde manifestó que solo ella se encuentra en el programa, y esto es por la falta de recursos humanos además el programa tiene una población de adultos mayores de 571 inscritos y solo se trabaja con el $10 \%$, es ahí la problemática donde la enfermera del servicio no puede acudir a la visita domiciliaria de todos los inscritos, aun teniendo una programación, no se logra cumplir con todos los adultos mayores. A la vez, en las entrevistas a las cuidadoras nos dieron recomendación de que haya más enfermeras en dicho programa para que haya un control de cada uno de los adultos mayores.

El cuidado de los pacientes es la esencia de la profesión de enfermería, el cual se puede definir como una actividad que requiere de un valor personal y profesional encaminado a la conservación, restablecimiento y autocuidado de la vida que se fundamenta en la relación terapéutica enfermera-paciente. Sin embargo, existen situaciones que influyen en el quehacer del profesional de enfermería, olvidando en algunos momentos, que la esencia de enfermería, es el respeto a la vida y el cuidado profesional del ser humano ${ }^{16}$.

El cuidado de los pacientes representa una serie de actos de vida que tienen por finalidad y función mantener a los seres humanos vivos y sanos con el propósito de reproducirse y perpetuar la vida, de tal forma, el cuidado es mantener la vida asegurando la satisfacción de un conjunto de necesidades para la persona (individuo, familia, grupo y comunidad) ya que, en continua interacción con su entorno, vive experiencias de salud.

Las investigadoras consideraron que la importancia de la presencia de enfermería en el programa, es de alto grado ya que tienen la capacidad de servir al individuo enfermo, promover la salud, realizar prevención de enfermedades a los adultos mayores, prolongar la vida del hombre y dar apoyo emocional al familiar. De acuerdo a las manifestaciones que nos brindaron algunas cuidadoras, hicieron mención que debe incrementarse el personal de enfermería para una mayor atención a los pacientes en sus domicilios, y exista mayor tiempo de visitas domiciliarias. Se expresa en los siguientes discursos:

“...Nosotros necesitamos la terapista, es necesario que vengan al domicilio porque mi mamá ya no se le puede trasladar como antes, simplemente PADOMI viene $y$ hace una consulta muy ligera y se va nada más. Creemos que es conveniente que vengan más tiempo y que vengan más especialistas como el cardiólogo, terapista, neumólogo, hematólogo y enfermería porque mi mamá tiene diagnóstico de hipotensión pulmonar secundaria crónica - severo. Para que le saquen sangre $y$ eso es lo que tiene de dificultad que no viene muchos especialistas..."(PC7)

“...Yo creo que debería venir el geriatra, porque llevar a la persona no se puede, justo uno solicita el servicio PADOMI porque es muy difícil estar llevando la persona, como son tan sensibles captan las enfermedades..." (PC9)

Las autoras encontraron en algunos comentarios de las entrevistas, que las cuidadoras expresan la importancia de contar con un médico geriatra dentro del programa, para brindar una atención directa al adulto mayor, de tal forma que la valoración sea integral y así poder identificar aquellos problemas susceptibles de los adultos mayores. Las autoras no pueden dejar de mencionar que un geriatra sería lo adecuado para el programa, pero se reconoce que el programa cuenta con un médico para la atención de dichos adultos mayores. 
La valoración geriátrica en la atención domiciliaria contribuye a dar respuesta a necesidades no cubiertas y poner de manifiesto discapacidades no reconocidas. Los pacientes más beneficiados de las visitas domiciliarias son los pacientes frágiles y de alto riesgo; especialmente los recientemente hospitalizados o los que eran considerados candidatos a cuidados de larga duración. El beneficio se observa en la disminución de la mortalidad, del riesgo de institucionalización y en la funcionalidad. La importancia de la atención domiciliaria como parte del sistema estructurado de niveles asistenciales para la atención en geriatría radica en el hecho de ofrecer continuidad de los cuidados a los pacientes geriátricos con discapacidad aguda o crónica que lo requieran ${ }^{15}$.

Por ello, la importancia de la formación en competencias sobre gestión de servicios de salud radica en formar médicos y enfermeras que puedan dirigir organizaciones que no tengan solo conocimientos, sino habilidades y aptitudes distintas, una actitud de trabajo en equipo, logro de consensos, capacidad de entablar relaciones más horizontales entre médicos, enfermeras y pacientes, promover una nueva actitud, no solo como paciente, sino como usuario de los servicios de salud 17 .

Como investigadoras vemos la falta de otras especialidades anexados al programa de atención domiciliaria siendo necesario, ya que de esta forma cubrirían las necesidades de los pacientes que lo requieran, haciendo hincapié especialmente en las terapistas, psicología, etcétera ${ }^{18}$. Se muestra a continuación en los discursos:

"...existe falta de tiempo dado a cada paciente, porque vienen una vez a la semana por así decirlo y es necesario que realicen las visitas más días, por ejemplo, la señora tiene escaras y se debe de curar dejando un día, pero viene un día a la semana y eso es un poco contagioso que se esté segregando sus heridas..." (PC2)
“...Debería ser quincenal porque su enfermedad de la señora es muy crítica, a veces está bien, a veces el medico viene cuando ella está bien uno quisiera que viniera 15 días cuando ella está en su momento de crisis para que la observe..." (PC8)

Desde los inicios de la medicina y durante su evolución y desarrollo, se ha conferido especial importancia a la relación médico-paciente, por ser la clave para el éxito en la gestión asistencial. La práctica de la medicina, combina la ciencia y la tecnología con la aplicación de conocimientos y valores. Esta combinación gira alrededor de la interacción médico-paciente, elemento necesario para que la acción del médico pueda intervenir en las necesidades del enfermo. Como fenómeno complejo, es capaz de conducirse por dos caminos completamente opuestos: sanar o hacer daño. Se muestra en los siguientes discursos:

“...Me gustaría que a mi mamá la revisen para hacerle una terapia porque lo necesita, una capacitación para que de repente para que nosotras mismas lo podamos hacer en los momentos más difíciles que ella requiere..." (PC7)

“...Quisiera que viniera una terapista, le hiciera unos masajes a la señora, sus músculos amanecen duros, yo como casi no se de masajes, yo trato de hacerle masajes, darle sus pelotitas para que juegue, pero daría gusto que viniera una terapista para que le haga esos ejercicios o una enfermera a ayudar..." (PC8)

La entrevista médica es la herramienta fundamental para obtener una anamnesis fidedigna y establecer una relación médicopaciente sólida, perdurable y productiva. La habilidad para conducirla debe aprenderse y debe perfeccionarse mediante el estudio, la práctica y la auto-observación. Una entrevista tiene valor terapéutico cuando el enfermo encuentra en el médico capacidades de respeto, interés, 
autenticidad y conexión. La habilidad para comunicarse eficientemente no solo consiste en saber expresarse, sino también en saber escuchar.

El médico, en principio, no puede comprometerse a curar a sus pacientes, pero está obligado a poner en práctica todos los cuidados necesarios, con vistas a lograr la curación del paciente, a mitigar el dolor o impedir eventuales complicaciones, incluso la muerte. Para el logro de ello, utilizará los conocimientos médico-científicos del momento y los medios a su alcance, conforme las condiciones específicas de modo, tiempo y lugar.

Si bien se conoce que el médico es el encargado del programa de atención domiciliaria, en tanto enfermería realiza diversas funciones que son necesarias para aquellos pacientes que lo necesiten. Así mismo, la relación enfermerapaciente es cuestión de cómo se lleva a cabo el proceso de visitas y la calidad de atención.

\section{RUTINIZACIÓN DEL EQUIPO DE SALUD DURANTE LA VISITA DOMICILIARIA}

El personal profesional de Enfermería debe tener claridad sobre su objeto de estudio y trabajo, es decir, de la importancia que tiene que sus objetivos, metas e intervenciones se enfoquen a fomentar y preservar la salud de la persona, de la familia y de la sociedad. Esto implica, entre otras concepciones, ubicar a la salud y su naturaleza, como el paradigma eje que guíe su ejercicio profesional ${ }^{19}$.

Se señala que por su propia naturaleza el equipo de salud está conformado por personal de diversas profesiones o disciplinas de las ciencias de la salud, que conocen los límites de su accionar y/o guías de actuación para realizar con mayor eficiencia y efectividad las intervenciones que le competen a cada uno y que en conjunto conducen al cumplimiento del objetivo del equipo al cual pertenecen.

Hasta el momento las funciones del equipo de salud giran en torno a satisfacer las necesidades de la población y a resolver las problemáticas en salud. En este sentido el profesional de enfermería, como integrante de ese equipo tiene sus funciones específicas que se realizan dirigidas a intervenir eficazmente con el individuo sano o enfermo en las diferentes etapas de la vida tanto en el área clínica como comunitaria, participando en la educación sanitaria al individuo, familia y comunidad y activamente en el desarrollo de programas de educación permanente ${ }^{20}$.

Cuando las participantes optan por aguantar y adaptarse a las condiciones desfavorables que les impone el medio, porque no encuentran alternativas o las oportunidades de empleo son escasas y no hacen nada para cambiar la situación; entran en un estado de conformismo y rutina de las tareas que poco o nada favorece la satisfacción como enfermera ${ }^{21}$.

Para las investigadoras, observamos en los diversos diálogos que la enfermera en su tiempo de visita domiciliaria realiza ciertas actividades como curación de heridas, cambio de sonda Foley, entre otros cuidados más. En cierta forma las cuidadoras aprecian y valoran el trabajo de la enfermera, ya que realiza su función con amabilidad, respetando la sensibilidad $y$ privacidad de los adultos mayores. Según los siguientes discursos:

“..La enfermera viene cuando tiene que cambiarle la sonda lo hace cada 15 días, la cambia, cura las heridas la revisa. La enfermera demora 10 min cuando no cambia sonda, solo la revisa y le ve la curación, cuando no le coloca sonda solo viene a revisar las heridas y realiza la limpieza de las úlceras y ella me dice cómo debo de hacerlo, por ejemplo, hoy vino cambió sonda, limpió todo y ya luego me explicó cómo debo de hacerlo yo..." (PC1)

"...La enfermera tiene su historia para la curación de heridas, todo proceso lo hace muy bien, lava, pone parches, y si es necesario colocarle algún antibiótico por la vía, también se le realiza. El tiempo en que 
demora la enfermera depende, porque ella tiene cuatro escaras, en la cadera derecha dos, una en la cresta iliaca y una en el sacro, se demora como $20 \mathrm{~min}$ o $30 \mathrm{~min}$ y tiene que lavarlo y todo..." (PC2)

En este contexto la Enfermería, además de aplicar de forma integral un conjunto de cuidados al individuo, la familia y la comunidad en el proceso salud-enfermedad, debe contribuir a que adquieran habilidades, hábitos y conductas que fomenten su autocuidado en el marco de una atención primaria integral y comunitaria que incluye la promoción, protección, recuperación y rehabilitación de la salud, y la prevención de la enfermedad 22 . Por otro lado, las cuidadoras manifestaron que los médicos vienen una vez al mes expresándolo en los siguientes diálogos:

\section{“...Muy buena la atención la doctora viene} una vez al mes cada 30 días, ellos nos dan la receta le toman la presión mira su corazón sus pulmones y le recetan y luego me voy al hospital al seguro a traer la medicina y me dan la medicina..." (PC1)

“...PADOMI es dado por el seguro social y consta de una visita al mes, el médico viene toma la presión, no hace más y receta. Digamos hace lo esencial como recentar pastillas para la presión, cremas para las escaras cosas así elementales..." (PC9)

"...la doctora viene cada mes, lo ve le deja su medicina, y después voy a reclamar al Naylamp, le dan medicamentos, le toma su presión también a mi cuñada, lo examina y después le toma la presión, y lo que nosotros queremos como son las pastillas, para las inyecciones..." (PC10)

En definitiva, como investigadoras vemos que la perspectiva de las cuidadoras en relación a la atención médica, es adecuada, ya que sienten la satisfacción con la visita del médico porque brindan la atención conforme a las necesidades a la que se somete el adulto mayor. Es necesario ampliar el tiempo de visita tanto medica como enfermera, para así mejorar las satisfacciones de la familia, el cuidador y el adulto mayor.

De igual manera, Rosenthal realizó una revisión sistemática que confirma que la Atención Médica en el Domicilio (AMD), como una fuente de atención centrada en el paciente, debe estar de la mano con la Atención Primaria en Salud (APS); se evidencia que la atención en casa es un avance en la salud de la sociedad. En la revisión se encontró que la Atención Médica en el Domicilio (AMD) mejora la percepción de la calidad por parte de los usuarios, por ende, genera mayor satisfacción con los servicios de salud y mejor aún, cuando los pacientes identifican su centro médico de Atención Primaria en Salud (APS). Así mismo, se documenta las ventajas de realizar la consulta médica del paciente en espacios diferentes al consultorio donde se permita la interacción con la familia. Además, se demuestra que la Atención Primaria en Salud (APS) tiene un alto grado de sensibilidad en cuanto a estilos de vida y menor utilización de los servicios de urgencias ${ }^{23}$.

Un asunto que es de primera importancia en la mente de muchos médicos es el factor tiempo. Quizás la principal duda que se presenta durante cualquier capacitación sobre comunicación en medicina es, si se puede lograr hacer todo lo que aquí se propone en el tiempo asignado habitualmente a las entrevistas médicas. Indudablemente, el tiempo es un factor importante y de hecho siempre apremia. Sin embargo, no se debe olvidar que las habilidades comunicacionales complementan y enriquecen aquello que ya se está haciendo en la anamnesis tradicional y, de hecho, una vez que las habilidades comunicacionales son manejadas adecuadamente, ellas no toman mucho más tiempo que el habitual. Por otra parte, sabemos que involucrarse en la relación o en el cuidado centrado en el paciente tomará más tiempo mientras el médico se encuentra en el proceso de aprender las habilidades. Una vez que las domina, los tiempos de consulta tienden a disminuir ${ }^{24}$. 
La verdadera eficiencia tiene que considerar el rendimiento clínico en términos de la precisión, de la calidad y del resultado final y no sólo del tiempo utilizado por consulta. Así mismo se puede evidenciar en los siguientes diálogos:

\section{“...El médico general lo evalúa le toma su} presión, lo evalúa sus pulmones le hace algunas preguntas de orientación como esta su lucidez y luego le receta la medicina, deja la receta y esperamos el lunes para recoger la medicina y hay médicos que se demoran unos 15 min a más tardar con conversar 20 min, hasta ahorita viene 3 médicos..." (PC3)

“...el doctor viene la revisa a mi abuelita, y le deja la receta, es un buen servicio... El doctor viene y le pregunta qué cosa le duele, bueno le dice si le duele algo, si necesita alguna otra cosita y ella le manifiesta algunas cosas y el doctor la receta y le deja, y le toma la presión arterial le receta y se va, aproximadamente se demora unos 20 $\min . . . "(P C 4)$

"...la doctora viene, le toma la presión en ese momento y nada más, después no, la doctora nos dice que nos hace falta. La doctora me anota para que yo reclame el medicamento la doctora no me deja o sea yo tengo que ir al Naylamp a recoger, se demora una media hora..." (PC7)

"...la doctora le controla la presión, pregunta acerca de sus problemas que ha tenido, y nada más. La doctora no demora más de 10 a 15 minutos, le toma la presión, la controla, le examina, no requiere mucho tiempo, pero si, le da medicina más bien ya cuando le falta alguna medicina y viene la doctora, le pido más medicamentos..." (PC1 1)

Las autoras plasmamos que, en cada entrevista se encontró la mención que hacen las cuidadoras respecto al tiempo de visita médica y pudimos resaltar que el tiempo es adecuado, sin embargo, esto podría prolongarse si el programa contara con un mayor número de personal de salud, para un mayor tiempo de atención, nosotras como investigadoras, vemos conveniente, que existan más especialistas en el programa de atención domiciliaria, porque la calidad de atención mejoraría y el tiempo de atención también.

A continuación, se presenta discursos, referidos a las interconsultas que brinda el programa de atención domiciliaria para los adultos mayores:

“...Bueno viene a eso de las 12, la examina le toma la presión, pide sus medicamentos para ver cómo va, nada más y si yo requiero una interconsulta el me la da, yo le pido y me da por eso le digo es bien atento bien amable, demora aproximadamente media hora, y viene una vez al mes, el médico si tiene que ser una vez al mes me da la receta para traerlo de la farmacia..." (PC5)

“...viene el médico, la chequea a la señora, le deja su medicina y le toma la presión, me pregunta su medicina, todo eso... Media hora, está el médico, la chequea y deja su receta y se va, se despide. Le toma la presión, le pregunta como esta, yo le contesto, me da los medicamentos, o si tiene una dolencia más, yo le digo, él lo observa y le vuelve a dar otra medicina..." (PC8)

"...La doctora cuando viene le pregunta cómo se encuentra, si tiene algún síntoma. $Y$ de ahí le toma la presión y luego le receta la medicina que es de todos los meses. La doctora los ve a los dos mis papas, se demorará unos 20 min..." (PC6)

Las autoras expresamos que los beneficios ofrecidos por el programa consisten en: la intervención de otros servicios; es decir, las interconsultas que genera dicho programa; los cuidadores en cada visita médica solicitan interconsultas para generar un mejor cuidado con otras especialidades. En aquellos casos que no encuentran un diagnóstico cierto para una patología, se hace necesario una interconsulta. 
Una comunicación efectiva promueve la colaboración entre el médico y el paciente, donde el foco de la entrevista no está centrado en el médico ni en el paciente, sino en la relación de ambos. Parte importante del aprendizaje de cualquier competencia es la definición clara que desglosa sus componentes: conocimientos, habilidades y actitudes. Hoy existe una base sólida de evidencia que aporta al conocimiento de cuáles son los componentes de una comunicación efectiva en medicina ${ }^{24}$.

Con una comunicación efectiva las molestias en el paciente disminuyen y aumentan su satisfacción. No se trata únicamente de mejorar los aspectos psicológicos de la atención. Aunque son pocos, existen estudios, incluyendo ensayos randomizados, que muestran que mejorando la comunicación en aspectos específicos también mejoran los resultados fisiológicos. Dos estudios independientes demostraron una mejoría en las glicemias de pacientes diabéticos cuando sus médicos aprendieron habilidades comunicacionales que les permitían centrarse más en el paciente.

Para las autoras el significado de rutinización, está relacionado a la costumbre o un hábito que se adquiere de forma determinada, en tanto el personal de salud del programa PADOMI cuando realiza la visita domiciliaria realiza diversos procedimientos que están a cargo de la enfermera como colocación de sonda nasogástrica entre otros procedimientos, y también la doctora realiza el control de la presión arterial entre más actividades.

Es triste y desalentador, ver como la enfermera por diversos aspectos rutiniza su labor, se acostumbra al dolor, a la muerte, es como si tuviera tal vez anestesiados sus sentimientos y emociones. Esta situación debe parar, los profesionales de la salud por ningún motivo podrán perder su sensibilidad, su poder humano ya que afortunadamente, para dicha de ellos, su labor siempre va a ir dirigida a cuidar un ser humano, no a una máquina, que se manipula sin pudor, sin consideración ${ }^{25}$.

\section{PERCEPCIÓN DE LA ATENCIÓN DOMICILIARIA POR PARTE DE LOS FAMILIARES}

Home Care menciona que los cuidados a domicilio designan de forma genérica un amplio abanico de servicios de apoyo para personas dependientes y con incapacidades. La denominación incluye desde servicios de ayuda para las actividades de la vida diaria y tareas domésticas que requieren una cualificación mínima, hasta cuidados profesionales que utilizan tecnologías sofisticadas. Esta modalidad de atención varía también en términos de la duración de los servicios: en unos casos se ofrecen por un breve periodo tras una hospitalización, mientras que en otros se trata de cuidados de larga duración requeridos a consecuencia de procesos crónicos invalidantes aunque la gama de cuidados que se ofrecen en el hogar incluye la asistencia médica, terapia ocupacional y fisioterapia, el interés se centra, mayoritariamente, en el análisis de los cuidados enfermeros a domicilio y en los servicios de ayuda domiciliaria, al ser éstos los que constituyen la base de los cuidados de larga duración ${ }^{26}$. Se aprecia en los discursos siguientes:

“...El programa si es bueno porque permite también que los atiendan en casa y es mejor para nosotros para ya no estar llevándolo, bueno yo le hice su gestión en PADOMI porque a mi mami yo la llevaba y tenía mucho trabajo en llevarla entonces hice su trámite de PADOMI..." (PC5).

"...Quisiera que no se acabe el programa, porque es bueno, ya que nos dan todo, la medicina eso, muy bien, la doctora es buena..." (PC10)

“...es un programa de bienestar hacia el adulto mayor que facilita sin el caso que el adulto mayor tenga alguna enfermedad o un mal, permita la curación, una atención continua por así decirlo para que tenga un bienestar de su salud en lo que le queda de vida, la disponibilidad que tiene para venir a 
curarlos en este caso y la disponibilidad sobre todo..." (PC2).

Las investigadoras opinan que la atención domiciliaria debe tener beneficios para el adulto mayor y la familia, es por eso que la atención se brinda en sus hogares para una mayor facilidad ya que la enfermera encargada del programa acude según programación a la visita domiciliaria, realizando una atención directa con el adulto mayor en el propio hogar siendo esto de mucha ayuda para las cuidadoras porque facilitan el cuidado mismo. Cabe mencionar que el programa no solo favorece a los adultos mayores con solo visitas domiciliarias, sino que apoya con los medicamentos que necesita el adulto mayor, siendo favorable y positivo para las cuidadoras, ya que solo necesitan acudir a farmacia y reclamar los medicamentos necesarios, y de tal forma se evidencia a tales cuidadoras conformes en el trato.

La asistencia sanitaria a domicilio se encargará de ayudar a aquellas personas que por sus propios medios no pueden acudir al centro de salud, por tanto, serán los profesionales los que tendrán que desplazarse a sus hogares. Debido a que la mayoría de los pacientes antes mencionados se encuentran incapacitados, el sistema sanitario ha implementado estrategias de actuación, principalmente desde la atención primaria ${ }^{27}$.

Ya que el cuidado de estas personas conlleva una importante carga de trabajo para los profesionales sanitarios y sus familiares, la red de servicios sanitarios debe adaptarse continuamente a las nuevas necesidades que demanda la población. Por ello, en las áreas del Sistema Nacional de Salud de las distintas comunidades autónomas, ya existen programas o protocolos que, con mayor o menor desarrollo en la práctica, centran la atención sanitaria en el domicilio de algunos grupos de pacientes o de individuos, proporcionándoles atención directa en el propio hogar mediante intervenciones específicas que favorezcan su permanencia e integración en su entorno habitual, ofreciendo la mayor comodidad posible dentro de su problema de salud.
Un equipo de Atención Domiciliaria lleva a cabo la asistencia de los cuidados físicos, psíquicos y sociales, prestados en el domicilio del individuo cuando este o la familia padece un proceso agudo o crónico y no tiene la capacidad o independencia suficiente para acudir al centro de salud. Las características de Atención Domiciliaria son: básica, de atención longitudinal y continuada, realizada en el domicilio del paciente, y cuyo núcleo de atención lo constituyen el enfermo y sus cuidadores, tanto familiares como informales. Su gestión parte de los profesionales de Atención Primaria atendiendo tanto procesos de enfermedades crónicas como agudos derivados del alta hospitalaria. Se expresa en los discursos:

“...Lo positivo es que nos ahorra el tiempo de trasladarlo a su consulta y eso para nosotros es valioso porque yo que trabajo y mi esposo que es una persona que ya tiene su edad también nos facilita a nosotros y así como también nos facilita la terapia..." (PC3).

"...Bueno en una parte que viene al domicilio
$y$ eso es lo que nos favorece a nosotros, porque eso de ir al seguro es un problema porque demoran horas, ellos son unas personas que tiene que ser atendidas, pero demoran bastante para atenderlo, y bueno en tu casa ya es diferente. Lo bueno es que viene al domicilio, la doctora lo examina y eso es lo único que sé. La atención es muy buena la doctora..." (PC6)

“...Lo positivo es que, uno ya no va al seguro, porque es una demora, y además su edad de la señora no permite estar llevándola, trayéndola, lo mejor es que el médico haya venido a chequearla mensualmente..." (PC8).

Es cierto que la existencia de un programa de atención domiciliaria favorece al adulto mayor, y conforme a eso las autoras toman en cuenta que estos programas deben crecer en beneficios para el adulto mayor y la misma familia, brindado un 
mayor número de especialidades dentro del programa en beneficio y mejora de la salud de aquellos inscritos en dicho programa. En opiniones rescatadas de las cuidadoras sienten que el programa es de gran ayuda porque no necesitan acudir al hospital para una atención médica, puesto que el propio médico realiza su visita a cada hogar según programación, y también no necesitan hacer largas colas de espera para una consulta o espera de atención y es por eso que las mismas cuidadoras se sienten conformes en los beneficios encontrados del programa de atención domiciliaria28.

Desde la perspectiva de calidad de vida percibida por el usuario, se ha constatado en las experiencias existentes, nacionales e internacionales, que el SAD (servicio de atención domiciliaria) reduce el sentimiento de, soledad, aislamiento y desprotección, ofrece seguridad a las personas en situación de dependencia y promueve una atención socio-sanitaria más continuada y conectada con el entorno asistencial ${ }^{29}$.

Los beneficios potenciales esperados del servicio de atención domiciliaria, social o médica, han de analizarse siempre desde la perspectiva conjunta de los usuarios finales en el hogar (personas en situación de dependencia, discapacidad o mayores y familiares), los profesionales (asistentes sociales, médicos de familia, especialistas, psicólogos, personal de enfermería, terapeutas ocupacionales, auxiliares de hogar) y los proveedores del servicio.

La atención a domicilio, es un recurso que permite proveer asistencia sanitaria para la resolución en el domicilio del paciente, problemas de salud, que por su situación de incapacidad física no puede desplazarse a un centro de salud, entendiendo que el nivel de complejidad de los problemas de salud a resolver, no requiere la hospitalización del paciente, la realiza el equipo de atención primaria y tiene características que la definen y la diferencian de la hospitalización en domicilio.

Tenemos entonces un conjunto de características a las cuales el profesional que brinda asistencia de urgencia en domicilio deberá enfrentarse y adaptarse, ya que formarán parte del desarrollo de este tipo especial de relación médico paciente. El profesional debe llegar a conocer al paciente, a su dolencia, y a un entorno con alto nivel de tensión y expectativa, de una manera ágil y rápida, para brindar una solución, todo en un ambiente que no es el hospitalario 30.

\section{Conclusiones}

Las personas cuidadoras en sus percepciones añaden que es la enfermera la responsable del programa de atención domiciliaria, en tanto evidencian la escasa participación del personal de enfermería. En las entrevistas realizadas se observó, que existe escasa participación debido a que la falta de coordinación por el hospital para gestionar un mayor número de personal de enfermería, teniendo como consecuencia la baja calidad de atención que debería realizarse para toda la población inscrita en dicho programa.

Las limitantes que se evidencian en el programa es el gran número de actividades que tiene la enfermera encargada, siendo la única que tiene toda la responsabilidad, y es ahí en donde no puede abarcar a cumplir con todos los adultos mayores inscritos, aun realizando una programación, se ve reflejada la limitante de profesionales enfermeros para el programa de atención domiciliaria.

El equipo de salud perteneciente al programa, cumple con lo que necesita cada adulto mayor en las diferentes visitas domiciliarias, en tanto se evidencia rutinización en todos los integrantes del equipo de salud, ya que cumplen con cada procedimiento asistencial y administrativo; descuidando el aspecto humano de la persona y, por consiguiente, la calidad de atención.

Los familiares de las personas adultas mayores, expresan que es favorable el programa de atención domiciliaria, ya que son muchos los beneficios encontrados, mejora la atención porque el médico acude al domicilio de cada persona, reciben medicamentos de forma gratuita gracias a la 
inscripción en dicho programa; entonces las personas cuidadoras sienten satisfacción cuando hablan del programa de atención domiciliaria.

\section{Bibliografía}

1. Organización Mundial de la Salud. Personas adultas mayores y familias. Perú: OMS; 2012. [consultado el día 15 de abril de 2016]

2. Ministerio de Salud. Lineamiento para la atención integral de salud de las personas adultas mayores. Informe técnico del MINSA. Lima: MINSA; 2006. [consultado el día 15 de abril de 2016]

3. Alvarado F, Diana C. Nivel de agotamiento que presentan los cuidadores familiares del adulto Mayor frágil. Centro de atención Del adulto mayor "tayta Wasi". Tesis doctoral. Universidad Nacional Mayor de San Marcos. Facultad de Medicina. Lima; 2014. (documento en línea:

http://cybertesis.unmsm.edu.pe/bitstream/c ybertesis/3526/3/Alvarado_fd.pdf)

[consultado el día 17 de abril de 2016]

4. Ferré-Grau C, Rodero-Sánchez V, Cid-Buera D, Vives-Relats C, Aparicio-Casals MR. Guía de Cuidados de Enfermería: Cuidar al Cuidador en Atención Primaria. Publidisa. Tarragona; 2011. [consultado el día 17 de abril de 2016]

5. Flores E, Rivas E, Seguel F. Nivel De Sobrecarga en el Desempeño del Rol del Cuidador Familiar de Adulto Mayor con Dependencia Severa. Cienc. Enferm; 2012. (documento en línea: http://www.scielo.cl/scielo.php?script=sci_ar ttext\&pid $=$ S071 7-

95532012000100004\&lng=es.http://dx.doi. org/10.4067/S0717-95532012000100004)

[consultado el día 19 de abril de 2016]

6. SAMPIERI y colaboradores. Metodología de la investigación: cuantitativa y cualitativa (4‥ed.). Mc Graw-Hill Interamericana. México; 2006. [consultado el día 9 de junio de 2016]

7. Bardin L. El análisis de contenido. 3era ed. Madrid: Akal S.A; 2002. [consultado el día 20 de junio de 2016]
8. Tello C, Gutiérrez P. Método y técnicas de análisis cualitativo. Sección De Posgrado De Enfermería- Escuela De Posgrado Universidad Nacional de Trujillo; 2009. [consultado el día 23 de junio de 2016]

9. Sgreccia E. Manual de bioética. En: Nureña M, Julia M. Liderazgo que ejerce la enfermería en el fomento de la salud familiar para el desarrollo comunitario. Valle hermosoMonsefú, 2010 Tesis doctoral. Universidad Católica Santo Toribio de Mogrovejo. Escuela de Postgrado. Chiclayo; 2012. [consultado el día 23 de junio de 2016]

10. Puchia C, Jara P. Enfermería y el cuidado domiciliario de los mayores en la era de la globalización. CrossMark. Chile; 2015. (documento en línea: http://ac.elscdn.com/S1665706315000597/1-s2.0S1665706315000597main.pdf?_tid=74e878bc-ade6-11 e6-a2a800000aacb35e\&acdnat $=1479511779 \_3 a f 4 d 6$ 94b638bcd7e161b51a5d13d24a) [consultado el día 19 de noviembre de 2016]

11. Solís K, Guevara G. Programa de visita domiciliaria del sistema de salud costarricense: lineamientos para su mejora. Rev. Enfermería Actual en Costa Rica, 29, 1 18. Costa Rica;2015 (documento en línea: http://dx.doi.org/10.15517/revenf.v0i29.19 671) [consultado el día 19 de noviembre de 2016]

12. Álvarez J. Las enfermeras comunitarias ante las necesidades de las personas en el siglo XXI. FAECAP; 2012. [consultado el día 19 de noviembre de 2016]

13. Mariscal M. La Enfermería sin límites y los límites de la Enfermería. 2012. [consultado el día 26 de mayo de 2017]

14. Organización Panamericana de la Salud. LA OPS/OMS destaca la necesidad de formar más personal de enfermería en América Latina y el Caribe. Chile: OPS; 2015. (documento en línea: http://www.paho.org/chi/index.php?option= com_content\&view $=$ article\&id $=624:$ la opsoms-destaca-la-necesidad-de-formarmas-personal-de-enfermeria-en-america- 
latina-y-el-caribe\&ltemid =1005) [consultado el día 26 de mayo de 2017]

15. De la Revilla L, Espinoza J. La atención domiciliaria y la atención familiar en el abordaje de las enfermedades crónicas de los mayores. Aten Primaria. En: MichueBohórquez E., Arana-Maestre C., OrtizSaavedra P., Chávez-Jimeno H., Varela-Pinedo L. Perfil clínico de los pacientes que ingresan al programa de atención domiciliaria de Essalud en el sur de Lima Metropolitana. Rev. Soc. Perú Med Interna. Perú; 2011. (documento en línea:http://www.medicinainterna.org.pe/revi sta/revista_24_1_2011/perfil-clinico-de-lospacientes-que-ingresan-a-padomi.pdf.)

[consultado el día 17 de abril de 2016]

16. Juárez-Rodríguez, P, García-Campos M. La importancia del cuidado de enfermería. Rev Enferm Inst Mex Seguro Soc, 17 (2): 109-111. Perú; 2009. (documento en línea: http://www.medigraphic.com/pdfs/enfermeri aimss/eim-2009/eim092j.pdf) [consultado el día 26 de mayo de 2017]

17. Cecilia M. La importancia de la formación por competencias para el personal de salud. Conexión Esan. Perú; 2012 (documento en línea:

http://www.esan.edu.pe/conexion/actualida d/2012/07/27/competencias-personalsalud/)[consultado el día 27 de mayo de 2017]

18. Sánchez D, Contreras Y. La relación médicopaciente y su importancia en la práctica médica. Rev Cubana Med Mil. Cuba; 2014; 43(4). (documento en línea: http://www.bvs.sld.cu/revistas/mil/vol43_4_ 14/mil11414.htm) [consultado el día 27 de mayo de 2017]

19. García M, Arana B, Cárdenas L, Monroy A. Cuidados de la salud: paradigma del personal de enfermeros en México - la reconstrucción del camino. Esc. Anna Nery [Internet]. 2009 June [cited 2017 May 23]; 13(2): 287296. Available from: http://www.scielo.br/scielo.php?script=sci_a rttext\&pid $=$ S1414-

$81452009000200008 \&$ Ing $=$ en. http://dx.do i.org/10.1590/S1414-81452009000200008.

20. De Tejada G. Enfermería en el equipo de salud. Enfermería y el equipo de salud; 2013. (documento en línea: http://clasesfundamentosdeenfermeria.blogs pot.pe/2013/01/funciones-del-equipo-desalud.html) [consultado el día 27 de mayo de 2017]

21. Dibábora E, Rossi C. La construcción de la identidad profesional en el sistema educativo y en el sistema prestador de la atención en salud. Temas de Enfermería Actualizados. 1998; 6(27): 9-15 [consultado el día 27 de mayo de 2017]

22. Bernat R. La enfermería en atención primaria. Análisis de la situación y propuestas de mejora. Catalunya, España; 2015. (documento en línea: http://www.mareablanca.cat/laenfermeria-y-la-atencion-primaria/) [consultado el día 22 de mayo de 2017]

23. Rosenthal T. The medical home: growing evidence to support a new approach to primary care. The Journal of the American Board of Family Medicine. 21(5), 427-440. España; 2011 . [consultado el día 22 de mayo de 2017]

24. Moore P, Gómez G, Kurtz S, Vargas A. La comunicación médico-paciente: ¿Cuáles son las habilidades efectivas? Rev Med Chile, 138: 1047-1054. Chile; 2010. (documento en línea:

http://www.scielo.cl/pdf/rmc/v138n8/art16. pdf) [consultado el día 22 de mayo de 2017]

25. Cerón Y. Aspectos presentes en la comunicación de la enfermera, la familia y de la institución en la Unidad de Cuidado Intensivo. Pontificia Universidad Javeriana; 2008. (documento en línea: http://www.javeriana.edu.co/biblos/tesis/enf ermeria/tesis52.pdf) [consultado el día 27 de mayo de 2017] 
26. Gonzalo E, Toral I, Ruiz C, Martín F, Morales J. Estrategias de mejora de la atención domiciliaria en Andalucía. Comisión Para El Desarrollo De La Atención Enfermera En El Servicio Andaluz De Salud. España; 2002. (documento en línea: http://www.indexf.com/lascasas/documentos/lc0228.pdf) [consultado el día 28 de mayo de 2017]

27. Alba P. Atención Domiciliaria: Relación Terapéutica De La Enfermera Con La Familia. Universidad Autónoma de Madrid. España: 2016. (documento en línea: https://repositorio.uam.es/bitstream/handle /10486/674832/diaz_paulete_albatfg.pdf?se quence $=1$ ) [consultado el día 28 de mayo de 2017]

28. Espinel-Bermúdez M, Sánchez-García $S$, Juárez-Cedillo T, García-González J, ViverosPérez A, García-Peña C. Impact of the program home care for the chronically ill for elderly: quality of life and hospital readmissions. Salud pública Méx [revista en la Internet]. 2011 Feb [citado 2017 Jun 05]; 53(1): 17-25. Disponible en: http://www.scielo.org.mx/scielo.php?script= sci_arttext\&pid $=$ S0036 $36342011000100004 \&$ lng $=$ es.

29. Fernández J. Guía Del Servicio De Atención a Domicilio. Ministerio De Sanidad, Servicios Sociales e Igualdad. España; 2010. (documento en línea: http://www.oiss.org/IMG/pdf/GUIA_SERVICIO _ATENCION_DOMICILIARIA_Prog-Ib-def-.pdf) [consultado el día 28 de mayo de 2017]

30. Diego K. La relación médico paciente en la urgencia domiciliaria. Revista Médicos. Argentina; 2013. (documento en línea: http://www.acudiremergencias.com.ar/larelacion-medico-paciente-en-la-urgenciadomiciliaria/) [consultado el día 28 de mayo de 2017] 